\title{
Chronic bullous disease of childhood and a paecilomyces lung infection in chronic granulomatous disease
}

\author{
J H Sillevis Smitt, J H W Leusen, H G Stas, A H Teeuw, R S Weening
}

\begin{abstract}
A 12 year old boy suffering from p67-phox deficient chronic granulomatous disease presented with a bullous skin disease and a lung infection with paecilomyces species. The histopathology of a bullous lesion showed subepidermal blister formation and microabcesses containing eosinophils in the dermal papillae. By direct immunofluorescence, linear staining of $\operatorname{IgA}$ at the dermal-epidermal junction was detected which confirmed the clinical diagnosis of chronic bullous disease of childhood (linear IgA dermatosis)

(Arch Dis Child 1997;77:150-152)
\end{abstract}

Keywords: chronic granulomatous disease; chronic bullous disease; paecilomyces; p67-phox

Chronic granulomatous disease (CGD) is a primary immunodeficiency due to a failure of polymorphonuclear leucocytes and monocytes/macrophages to produce reactive oxygen products (for example, hydrogen peroxide, oxygen radical). Several subtypes of CGD have been identified with distinct molecular defects. Based on the enzyme system that produces oxygen radical (NADPH oxidase), which consists of several components, the following CGD variants are known ${ }^{1}$ :

- The X linked variant, in which the large subunit (gp91-phagocytic oxidase (phox)) of the membrane bound cytochrome- $b_{558}$ is affected, or

- Three autosomal recessive variants, in which either the small subunit (p22-phox) of cytochrome- $b_{558}$ is affected or one of the cytosolic proteins $\mathrm{p} 47$-phox or p67-phox.

In most cases of cytochrome- $b_{558}$ affected variants, both subunits of this heterodimeric protein are absent, and in all cases of defects in the cytosolic proteins the affected protein is absent. The p67-phox variant is very rare.

In CGD, autoimmune diseases have been described. In particular, the occurrence of discoid lupus erythematosus is a well known phenomenon. ${ }^{2}{ }^{3}$

Chronic bullous disease of childhood is an autoimmune blistering disease in children with an adult counterpart (linear IgA dermatosis), characterised by the presence of a linear band of IgA at the dermal-epidermal junction. As far as we know, chronic bullous disease of childhood has never been reported in primary immunodeficiency disorders. We observed a boy with p67-phox deficient CGD, who pre- sented with chronic bullous disease of childhood and a paecilomyces lung infection, rarely seen in CGD.

\section{Case report}

In 1994 a 12 year old boy from Pakistan arrived in the Netherlands. He was the eldest son in a family of four living children, four others died at an early age in Pakistan of unknown cause. His father and mother were cousins. The patient had suffered from skin and lung problems during the past five years. The skin problems consisted of small itching lesions spread over the entire body, starting as blisters and changing rapidly into crusts. The pulmonary disease manifested itself as a chronic cough and dyspnoea during exercise. He was chronically tired and failed to thrive. Treatment with antibiotics (sulphamethoxazole) resulted in short term remissions of lung and skin problems. At the time of his first visit to our department he was taking flucloxacillin. His medical history showed no other problems.

At physical examination a pale, slender, slightly tachypnoeic 12 year old boy was seen. Respiration rate was $30 / \mathrm{min}$, pulse $92 / \mathrm{min}$, blood pressure $85 / 60 \mathrm{~mm} \mathrm{Hg}$; his body length was $1.36 \mathrm{~m}(<3 \mathrm{rd}$ centile on European growth charts), on hand radiography his bone age corresponded to the age of 10 years, and body weight was $25.5 \mathrm{~kg}$ ( $<3 \mathrm{rd}$ centile). A disseminated dermatosis was seen, consisting of erythematous lesions, some with central or peripheral crusting and others with central or peripheral blistering (fig 1). Some lesions showed a garland configuration. Neither scarring nor milia were seen. The eyes showed conjunctival injection and were watery. In his neck several enlarged lymph nodes were palpable. The chest movements were symmetrical. On auscultation, coarse crepitations were heard over all lung fields. Liver and spleen were normal on physical examination. Clubbing of the nails of the hands and feet was present; the joints were normal.

\section{FURTHER INVESTIGATIONS}

General

Erythrocyte sedimentation rate was $45 \mathrm{~mm} /$ hour, haemoglobin $6.9 \mathrm{mmol} / 1$, packed cell volume 0.35 , platelet count $482 \times 10^{9} / 1$, white cell count $10.3 \times 10^{9} / 1$, normal differentiation, eosinophils $310 \times 10^{6} / 1$, mean corpuscular haemoglobin 1.40 fmol (normal range $=1.5-$ 1.88), mean corpuscular haemoglobin $70 \mathrm{fl}$ (normal range $=77-96)$, serum iron 5.8 


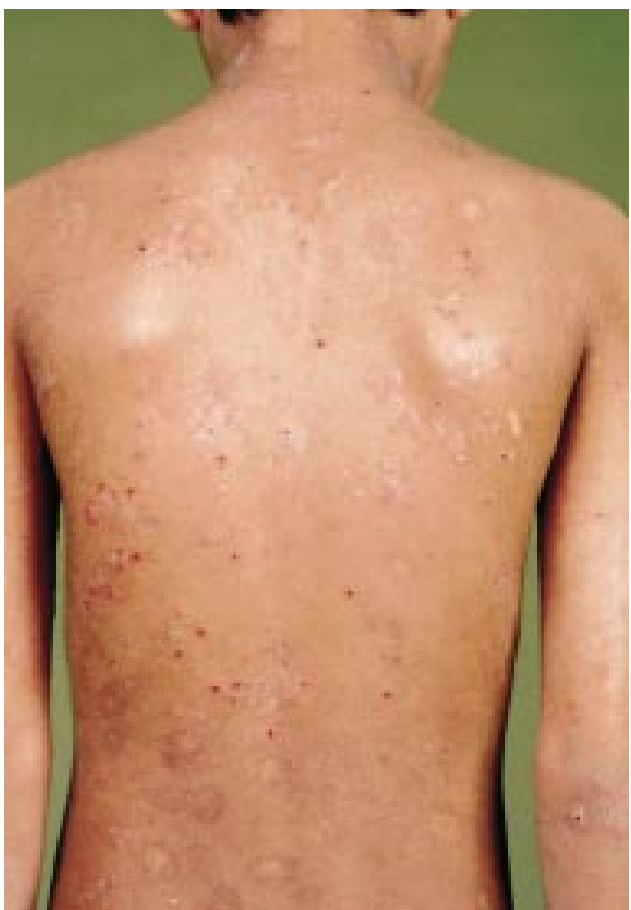

Figure 1 Vesicular lesions on the trunk, around a central crustous area.

$\mu \mathrm{mol} / \mathrm{l}$, and total iron binding ability 65.5 $\mu \mathrm{mol} / \mathrm{l}$. Arterial blood gas, electrolytes (sodium, potassium, calcium, phosphorus), liver function (aminotransferases, coagulation), and renal function (urea nitrogen, creatinine) were normal. Antinuclear antibodies, viral and aspergillus serology were all negative.

\section{Skin}

Bacterial culture of a blister was negative. Histopathology of a bullous lesion showed subepidermal blister formation. In the superficial dermis a mixed inflammatory infiltrate containing eosinophils was found. Microabcesses in the papillae contained eosinophils. By direct immunofluorescence of perilesional skin, a linear staining with IgA at the dermal-epidermal junction was detected. In addition, focal granular staining with IgM was seen. Indirect immunofluorescence (substrate: guinea pig lip) showed IgA staining of the basal layer of the epidermis in a dilution of $1: 320$.

\section{Lungs}

A chest radiograph showed widespread interstitial changes, central as well as peripheral. A computed tomogram of the lungs showed, in addition to the radiography, consolidations and thickening of the bronchial wall. In a lung biopsy specimen an interstitial fibrosis was seen, predominantly in the upper lobe. Moreover, several granulomata with giant cells were present. In a mycotic culture from lung biopsy specimens a paecilomyces species was detected. Cultures for bacterial, mycobacterial, viral, and parasitic pathogens were negative. The pulmonary function test indicated a severe restrictive pulmonary function disorder with a diffusing capacity for carbon monoxide of $22 \%$ of the normal value.
Gastrointestinal tract

A duodenal biopsy specimen revealed normal villi without any suspicion for coeliac disease.

\section{Immunological examinations}

These revealed slightly increased concentrations of immunoglobulins; he was housedust mite positive by radioallergosorbent test.

\section{Phagocyte functions}

The neutrophils showed normal chemotaxis, phagocytosis, and degranulation; the generation of oxygen metabolites was severely impaired. The child was found to have p67-phox deficiency using a cell free oxidase assay and western blotting. $^{4-7}$

\section{Clinical course}

Initially prednisone treatment was started because of suspicion of an extrinsic allergic alveolitis in combination with the autoimmune chronic bullous disease of childhood. The skin blisters disappeared but the pulmonary status remained unchanged. As soon as immunological test results were known, showing a defect in superoxide production of the granulocytes (compatible with the diagnosis of CGD) and the culture of the lung biopsy yielding a paecilomyces species, the oral prednisone treatment was stopped. Amphotericin B was given for four weeks followed by itraconazole. At withdrawal of the oral corticosteroids, the itch reappeared as well as erythematous papules and vesicular lesions. This eruption disappeared on local treatment with triamcinolone acetonide $0.1 \%$ in a lipophilic ointment. Besides the antifungal treatment, trimethoprim-sulphamethoxazole prophylaxis as well as interferon gamma treatment were started as part of the CGD management. The lung function remained stable. Because of the very poor nutritional state, nocturnal feeding by a nasogastric tube was given.

\section{Discussion}

Less than $2 \%$ of all known CGD patients lack the cytosolic p67-phox protein. Thus, clinical experience with this type of CGD is very limited.

In CGD, the most often encountered fungal lung infections are caused by aspergillus species. Paecilomyces infection in CGD has only been reported once before. ${ }^{8}$ In this patient, treatment with amphotericin B and later itraconazole was given with success. In our patient too, good results with this treatment were obtained.

Bullous autoimmune diseases in patients with a primary immunodeficiency have been reported sporadically. We found only one such case report, concerning an 8 month old girl with thymic hypoplasia, autoimmune haemolytic anaemia, and juvenile pemphigoid. ${ }^{9}$ Chronic bullous disease of childhood is a disease of unknown origin. In $38 \%$ of cases the dermatosis is preceded by a prodromal illness, such as an upper respiratory tract infection. ${ }^{4}$ In the patient described, it is not clear whether the lung disease preceded the start of the skin problems. Oxygen metabolites are necessary 
for the killing of bacteria and fungi. In suboptimally functioning granulocytes, biologically active remnants of digested cells may persist and act as neoantigens. These antigens may enhance the induction of autoimmune processes, and in this case may have led to the chronic bullous disease of childhood. Whether the presence of the paecilomyces infection in the patient presented is the origin for antigens that have led to IgA antibodies directed against the basement membrane substance is not clear. In linear IgA dermatosis in adults, it was found that the neutrophils of patients show an enhanced production of oxygen radicals. Moreover, neutrophils from healthy controls can be stimulated to higher oxygen radical production by serum from linear IgA patients. ${ }^{10}$ It has been suggested that oxygen radicals play a part in the tissue injury observed in linear IgA dermatosis. In CGD, oxygen metabolites cannot be produced. Our patient shows that without these metabolites the development of linear IgA dermatosis is still possible.

1 Roos D. The genetic basis of chronic granulomatous disease. Immunol Rev 1994;138:121-57.
2 Sillevis Smitt JH, Weening RS, Krieg SR, Bos JD. Discoid lupus erythmatosus-like lesions in carriers of X-linked chronic granulomatous disease. Br f Dermatol 1990;122: 643-50.

3 Sillevis Smitt JH, Weening RS, Krieg SR, Bos JD. Discoid lupus erythmatosus-like skin changes in patients with autosomal recessive chronic granulomatous disease. Arch Dermatol 1990;126:1656-8.

4 Roos D, De Boer M. Purification and cryopreservation of phagocytes from human blood. Methods Enzymol 1986;132: phagocy

5 Bolscher BGJM, Van Zwieten R, Kramer IJM, et al. A phosphoprotein of $M_{r} 47,000$, defective in autosomal chronic granulomatous disease, copurifies with one of two soluble components required for NADPH: $\mathrm{O}_{2}$ oxidoreductase activity in human neutrophils. F Clin Invest 1989;83:75763.

6 Leusen JHW, De Boer M, Bolscher BGJM, et al. A point mutation in gp91-phox of cytochrome $b_{558}$ of the human NADPH oxidase leading to defective translocation of the cytosolic proteins p47-phox and p67-phox. F Clin Invest 1994;95:2120-6.

7 Verhoeven AJ, Leusen JHW, Kessels GCR, et al. Inhibition of neutrophil NADPH oxidase assembly by a myristoylated pseudosubstrate of protein kinase C. $f$ Biol Chem 1993;268:18593-8.

8 Williamson PR, Kwon-Chung KJ, Gallin JI. Successful treatment of paecilomyces varioti infection in a patient with chronic granulomatous disease and a review of paecilomyces species infections. Clin Infect Dis 1992;14:1023-6.

9 Bloomfield S, Stockdill G, Barnetson RSC. Thymic hypoplasia, autoimmune haemolytic anaemia and juvenile pemphigoid in an infant. Br f Dermatol 1982;106:353-5.

10 Niwa Y, Sakane T, Shingu M, et al. Neutrophil-generated active oxygens in linear IgA bullous dermatosis. Arch Dermatol 1985;121:73-8. 